machinery silly, or mature?

There is little chemical and no biological detail in this book This lack of detail produces a screen on which the authors project a set of fantasies, many of which are of interest and worth considering, but what actually happens will likely be quite different to what is imagined here.

\section{References}

1. Healy D. Psychiatric drugs explained. Edinburgh: Churchill Livingstone;
2016. Chapter 16.

2. Healy D, Le Noury J, Mangin D. Links between serotonin reuptake inhibition pregnancy \& neurodevelopmental delay spectrum disorders. Int J Risk Saf Med. 2016 Sep 17; 28: 125-41.

3. Ames BN, Profet M, Swirsky Gold L. Medical Sciences. Dietary pesticides (99.99\% all natural). Proc Natl Acad Sci USA. 1990 Oct;87: 7777-81.

4. Healy D. The crisis in Cochrane: Evidence Debased Medicine. Indian J Med Ethics. 2019 Jan-Mar;4(1) NS: 52-4.doi:10.20529/JMME.2018.091

5. Healy D, Mangin D. Clinical judgments, not algorithms, are key to patient safety - an essay by David Healy and Dee Mangin. BMJ. 2019;367: 15777. doi: 10.1136/bmj.I5777.

\title{
Ethics and the vaccine wars
}

\section{DONALD W LIGHT}

Peter C Gotzsche, Vaccines: truth, lies and controversy, People's Press, 2020, 201 pgs, ISBN: 9788770368933, Kindle: Rs 1,426.

In his controversial but well-prepared style, Peter Gotzsche has jumped into what feels like the vaccine wars between the "vaccine advocates" who think that every vaccine should be taken by everyone as a vital part of preserving the public's health and the "vaccine deniers", who are characterised as believing vaccines are a dangerous kind of poison that government officials want to inject into the bodies of babes. Readers will find the book informative, interesting, and clear about what the real issues are. Gotzsche has a readable, informal style that students and readers will enjoy. $\mathrm{He}$ is a tough-minded sceptic who has been professionally involved in most of the chapters. Gotzsche is a physician and professor of clinical research design and analysis in trials, in Denmark. $\mathrm{He}$ is the author of many books and articles on health policy, often critical of the pharmaceutical industry for its bias, misinformation, and lack of transparency. He previously authored Deadly medicines and organized crime: How Big Pharma has corrupted healthcare, about drug companies "where cheating with clinical trials and in marketing is common and has led to hundreds of thousands of deaths." I wrote a review of this carefully researched book.

Vaccines consists of five substantial chapters on the conflicting messages about vaccines, vaccines for measles, mandatory vaccines, influenza vaccines, and controversies around HPV vaccine, in all of which ethical issues abound. They are followed by four brief chapters on Japanese encephalitis,

\footnotetext{
Author: Donald w Light (lightdo@rowan.edu), Professor of Comparative Health Policy and Psychiatry, Rowan University School of Osteopathic Medicine, Stratford, New Jersey, USA; Faculty Affiliate, Division of Medical Ethics, New York University, New York, USA.

To cite: Light DW. Ethics and the vaccine wars. Indian J Med Ethics. 2020 AprJun;5(2) NS: 165-6. DOI: 10.20529/JMME.2020.033.

Published online on April 4, 2020.

Manuscript Editor: Sanjay A Pai

OIndian Journal of Medical Ethics 2020
}

childhood vaccination programmes, and other vaccines, with a short three-page conclusion. The text runs into 187 pages of large type, followed by 23 pages of references. There is no table of contents or index, no preface or introduction. The chapters are well organised with good section heads.

All vaccines have risks and possible harms, Gotzsche writes. The question is whether a given vaccine is more beneficial, as many are. "It is unwise and unscientific to be universally against vaccines," he writes. "It is like being against all drugs or all people." The vaccine wars, however, mark all doubting or hesitating parents as "anti-vaxxers" and lump them together with the tiny percent of the population who oppose any vaccine. This cuts off intelligent conversation or consideration of those who have concerns about the industry-controlled trials and industry-produced evidence overseen by the Food and Drug Administration (FDA) or the European Medicines Agency (EMA), which are themselves funded by companies to review their evidence. Children get swept up in these battles so that unvaccinated children are now called "anti-vaccinated" children. Politicians get celebrated or vilified for raising questions about a given vaccine.

This battlefield is worth keeping in mind, because according to the vaccine policy center at New York University (1), there are 270 more vaccines in the pipeline! Imagine that 10 percent of these 270 vaccine-candidates get approved as "safe and effective." Parents and other adults will be faced with 37-45 vaccines, more than double the number now. Currently, 1018 vaccines are mandatory for infants and small children, depending on the country. But some countries, like the UK and Denmark, have no mandated vaccines, and a comparative study found no clear link between vaccine mandate and uptake. Vaccine culture varies a lot, led by an emphasis in the US. on mandating vaccines and by the Centers for Disease Control (CDC), which serves as an industry-funded spokesperson for the industry's point of view.

Driving the sprawling research enterprise that is developing 270 new vaccines is a massive pharmaceutical industry and the high prices that patented vaccines now frequently command. Prices 50 times manufacturing costs are not uncommon. 
"Beneficial" is a relative term, and vaccines are already being put in use that are only partially effective or for a limited time. The meanings of the term, "vaccine" are being stretched too, making the ethics of vaccines ever more complex.

Gotzsche is an empiricist and emphasises getting the facts right and paying attention to the science. Then there needs to be ethical consistency when two similar cases are treated differently. Third, we need to decide whether to weigh the deontological perspective about duties to autonomy higher or lower than the utilitarian goal of striving for the greatest good for the greatest number of people.

Gotzsche distinguishes between three kinds of paternalism: genuine paternalism, like parents caring for their children; solicited paternalism, when we ask another to decide for us; and unsolicited paternalism, when action is taken without permission. Healthcare is rife with unsolicited paternalism, "busybodies who love telling others what they should do, even when no one asked them..."

Gotzsche opposes forced vaccination as much as he opposes forced incarceration of people regarded as mad and dangerous. "We should avoid mandatory vaccination even if it is only indirect like prohibiting access to school for unvaccinated children, which can stigmatize them and might handicap their possibilities in life..." (Chap 3). However, "We have duties toward each other and by refusing vaccinations, the parents increase the risk that their children - and themselves as they made the decision - will harm other people." (Chap 3). Free riders are unethical.

Concerning influenza, Gotzsche writes that vaccination against it seems obvious but is not. Flu-like symptoms are far more common than influenza, and the vaccine does not reduce the number being hospitalised. Gotzsche's chapter on influenza vaccines focuses on distorted and doctored trials and the bewildering confusion of official information about them. The WHO hired experts paid by the manufacturers to write guidance. "The CDC website is a treasure trove of misinformation, even worse than what I have seen on drug company websites." He details examples and flawed examples of modelling projections. Gotzsche quotes the Oxford authority Tom Jefferson, saying "Influenza prevention has become an industry fueled by poor science and propelled by conflicted decision makers....Scaring people justifies evidencefree policies..." (Chap 4)

Gotzsche's details about the swine flu pandemic are sobering. Good data about safety is lacking. Regarding the requirement that healthcare workers be vaccinated, Gotzsche questions the ethics based on lack of evidence that it avoids hospitalisations and the paucity of evidence to support this utilitarian mandate. Canadian researchers found "no valid evidence to support the hypothesis that vaccinating healthcare workers protects patients from influenza." In addition, "Vaccination may provide staff with a false sense of security, which might reduce their level of handwashing" and increase patient risk.

While vaccination against measles gets a strong endorsement based on the evidence, the evidence for HPV vaccines is incomplete, biased, and troubling*. Gotzsche then tracks the EMA's responses and concludes it did a poor job assessing harms of the vaccine. A review suggests that "contrary to EMA's reassuring messages, adjuvants are harmful..." Merck minimised testing for safety of Gardasil and had no placebo controls. "Merck's reporting of serious adverse reactions is extremely misleading," he writes "Not a single trial was truly placebo (i.e. saline) controlled." "Because the HPV vaccines and their adjuvants had similar harms profiles, the manufacturers and regulators concluded that the vaccines are safe. This is like saying cigarettes and cigars must be safe because they have similar harms profiles." (Chap 5). At the EMA, "all mention of the safety concerns had been scrubbed." In the UK, spontaneous reports of adverse reactions from HPV vaccines "exceeded by far those for any other vaccine or combination of vaccines."

Gotzsche concludes that "It is unbelievable that [the] FDA let Merck get away with information that is so misleading..." He details how the conflict-ridden EMA was no better. He then turns to the Cochrane review of HPV vaccines as incomplete and flawed, like mistakenly calling active comparators placebos. Gotzsche led a systematic review of HPV vaccine trials and Cochrane's flawed report. For girls and women, he concludes that screening is most effective, complemented by vaccination.

One wishes Gotzsche had filled out the four very short chapters. In his brief chapter on childhood vaccination programmes, he points out how little we know about what sequence order is best and about vaccine interactions. Both are likely to have increasing relevance as more vaccines are approved. Perhaps we can look forward to an expanded second edition.

*Corrigendum: A sentence was deleted here on the request of the author of the review

\section{Reference}

1. NYU Langone Health Magazine. NYU Langone's new vaccine center takes aim at global health threats. nyulangonehealth.org. 2019 Spring [cited 2020 Apr 2]. Available from: https://nyulangone.org/news/nyulangones-new-vaccine-center-takes-aim-global-health-threats 